\title{
Paper Measurement of Electromagnetic Field Component Emissions as a Precursor of Emerging Hazard in Coal Mines
}

\author{
Remigiusz Mydlikowski ${ }^{1}$ and Krzysztof Maniak ${ }^{2}$ \\ ${ }^{1}$ Wroclaw University of Science and Technology, Wroclaw, Poland \\ ${ }^{2}$ National Institute of Telecommunications, Warsaw, Poland
}

https://doi.org/10.26636/jtit.2019.135419

\begin{abstract}
The article presents the results of research concerning electromagnetic (EM) field emitted by rocks during the process of their destruction. The paper describes a measuring stand designed for subjecting rock samples to axial crushing. During the destruction of rock samples, both components of the electromagnetic field emitted by the rock were recorded. The paper presents the results of research on hard coal samples, presenting the obtained time domain waveforms and frequency spectrum of the emitted EM field components. Further potential uses of tests concerned with EM emitted by rocks are determined. Applications registering EM field in mine environments may provide an early mine collapse warnings.
\end{abstract}

Keywords—coal mining, electromagnetic field, rock destruction, subsurface geophysics.

\section{Introduction}

Seismoacoustic testing is the standard method used for assessing the stresses within coal rock. Rock stresses [1] are recorded by means of a network of geophones located at locations that are of critical importance for mining operations. Proper interpretation of the results of these measurements makes it possible to determine those active mine zones that face the threat of destruction. However, despite numerous efforts made by personnel in charge of safety, tragic catastrophes still occur.

Numerous new research methods are being introduced. One of them is the measurement of anomalous electromagnetic fields that accompany increased mechanical stresses present in the rock jacket, e.g. hard coal, that is navigated through. The first reports on anomalous electromagnetic fields accompanying earthquakes date back to the late 1800s. It was in 1890 that Milne published a report on anomalous magnetic and electrical phenomena accompanying earthquakes. The advance in technology that took place in the late 2000s made it possible to use this phenomenon in practice to predict disasters related to increased levels of stress in rock material, occurring during earthquakes, landslides and mining disasters. In the last fifty years, intense work was performed on the theory and on its practical applications.

For example, in 1960, an increased level of electromagnetic emissions was observed at the frequency of $28 \mathrm{MHz}$ a few days before the earthquake in Chile [2]. Similar phenomena were observed before the earthquake in Indonesia in 1996, some 1,200 kilometers from the epicenter [3]. In practice, spontaneous electromagnetic emissions generated by rocks are more frequently used in hard coal mines.

In recent years, several devices have been developed for measuring electromagnetic field emitted from rocks in which stresses occur. One of them is a portable device with a ferrite antenna, known as the Cerescope [4]. The device enables continuous recording of the magnetic component of the electromagnetic field, with its maximum sensitivity achieved at $12.8 \mathrm{kHz}$. The frequency band should be 5 to $50 \mathrm{kHz}$. Another portable device is the KBD5 system, consisting of a directional antenna used as a sensor, connected to an acquisition and recording device [5]. Here the frequency band is wider: $1-500 \mathrm{kHz}$. A similar device - FJV99 - sensing the magnetic component at $14.5 \mathrm{kHz}$ only, was developed for the Czech Academy of Sciences [6].

Research concerned with EM emissions generated by rocks faces the problem of interferences caused by power grids, human settlements and traffic. To cope with this, sophisticated algorithms have been developed allowing to obtain weak signals from rocks against the background of strong interfering signals [7].

However, no details concerning the devices used for recording electromagnetic field emissions, whose sensors are located at the critical points throughout the mine, are available. What is worse, researchers do not share the parameters of EM field components, providing only the output voltages of the measuring devices, or conventional units. 


\section{Generation of EM Field}

The primary mechanisms initiating spontaneous electromagnetic fields generated under the influence of stresses inside rock formations include the piezoelectric effect and micro-cracks in the rock structure.

The piezoelectric effect is observed in anisotropic dielectric media with a regular crystallographic structure. It occurs mainly in the case of rocks containing quartz crystals in their structure, such as granite or sandstone. The piezoelectric phenomenon occurs also in minerals with dielectric and semiconductor properties, such as selenium, tellurium, greenockit and zinc.

The dominant phenomenon here is the reverse piezoelectric phenomenon described by [8]:

$$
D=e \cdot S+\varepsilon^{\varepsilon} \cdot E,
$$

where: $D$ - electric displacement, $E$ - intensity of electric field, $S$ - strain, $e-$ piezoelectric constant, $\varepsilon^{\varepsilon}-$ dielectric permittivity for constant strain.

Research concerning electromagnetic emissions generated by piezoelectric active samples has resulted in observations of a wide frequency spectrum [9].

The second effect is caused by micro-cracks in the rock structure. It is a natural phenomenon, as microcracks and fissures are created in the rock subjected to a destructive force and, thus, to increased internal stresses. The fissures can be considered capacitors whose covers are loaded with dampened vibrations. The model of a crack propagating along the $\mathrm{x}$ axis, where $P_{0}$ is the stress force, is shown in Fig. 1 [10].

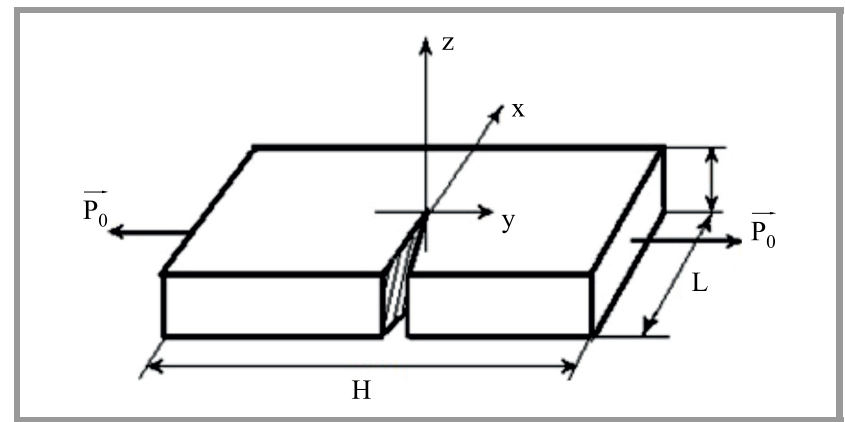

Fig. 1. The process of rock structure micro-crack formation.

The formula linking the electric field strength $E$ with the characteristic of the micro-crack created is [10]:

$$
\Delta E-\nabla \operatorname{div} E-\frac{\varepsilon_{i j} \partial^{2} E_{j}}{c^{2} \partial t^{2}} e_{i}=\frac{1}{\varepsilon_{0} c^{2}} e_{i k l} \frac{\partial^{2} U_{k l}}{\partial^{2}} e_{i}
$$

where: $U_{k l}$ - components of the deformation tensor, $E-$ intensity of electric field, $c$ - the speed of light, $\varepsilon_{0}-$ permittivity of free space, $\varepsilon_{i j}$ - components of dielectric tensors of the crystal, $e_{i k l}-$ components of piezoelectric tensors of the crystal, $e_{i}-$ unit vector of the crystallographic system.
Another phenomenon, known as turbulent flow of liquid through rock micropores, plays an important role in rocky materials with a porous structure. The liquid (water, etc.), subjected to stresses and vibrations of the structure, flows through the capillaries in a turbulent way, at the speed of $\overrightarrow{v_{c}}$. This flow takes place in the $\overrightarrow{B_{E}}$ geomagnetic field of the Earth [11]. Then, on each electron of charge $e$, the Lorentz force acts, having the following value:

$$
\overrightarrow{F_{L}}=e \cdot \overrightarrow{v_{c}} \times \overrightarrow{B_{E}}
$$

This force contributes to the orderly motion of electrons by producing an $E$ field:

$$
\vec{E}=\overrightarrow{v_{c}} \times \overrightarrow{B_{E}}
$$

This phenomenon also occurs in rocks devoid of quartz crystals and in wet soils conducive to the development of landslides.

\section{Measurement Setup}

The measurement system consists of a $450 \mathrm{kN}$ hydraulic press, as well as separate field recorders $E$ and $H$ (Fig. 2).

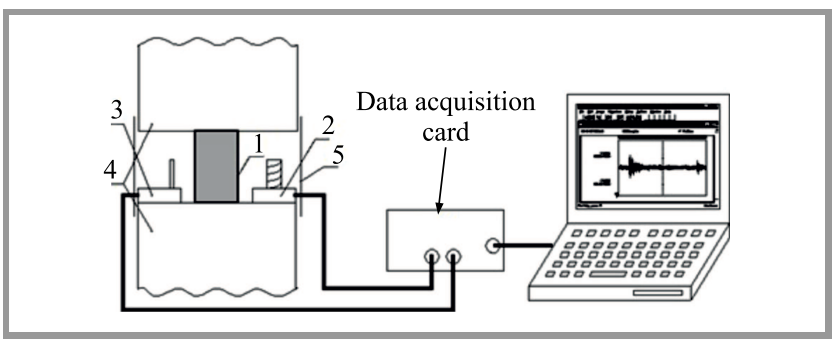

Fig. 2. Setup for recording EM fields generated rock samples subjected to destructive loads: 1 - test sample, 2 - magnetic field sensor, 3 - electric field sensor, 4 - hydraulic press, 5 - electromagnetic screen.

Here, the EM field generated by the rocks is sensed in a near-field, where it is difficult to establish a clear relationship between $E$ an $H$ components. Therefore, it is necessary to use separate sensors placed at the height of $2-3 \mathrm{~cm}$, on rubber damping pads, as this counteracts the so-called microphone effect. The setup allows to measure EM components within the frequency range of 0.1 to $100 \mathrm{kHz}$.

Next, the data acquisition card with relevant software converts the analog signals coming from sensors into a digital form, enabling further observations to be performed on a computer screen and recorded.

Both sensors are battery-powered, which eliminates interference caused by the power supply network. In addition, to avoid interference, the entire test stand is shielded with a $1 \mathrm{~mm}$, grounded sheet of copper. To assess shielding efficiency, an experiment measuring external interference was conducted. It was carried out in room that is located at a considerable distance form strong EM interferences 
(a)

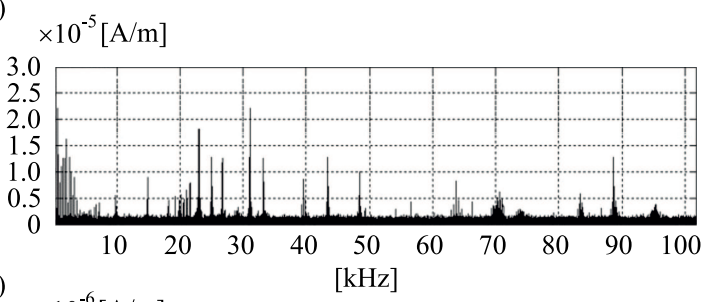

(c)

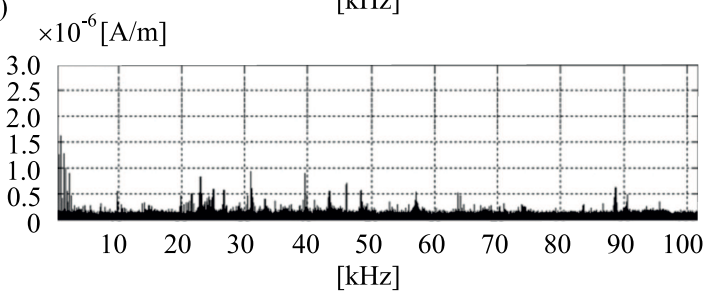

(b)

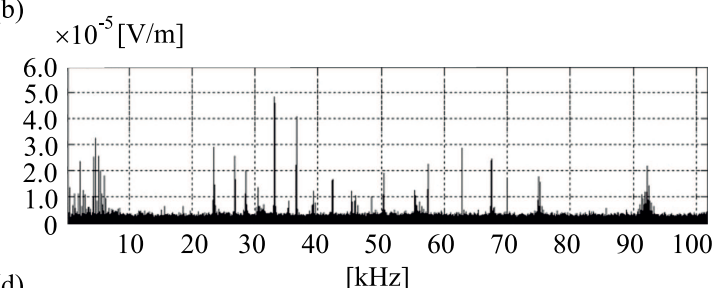

(d)

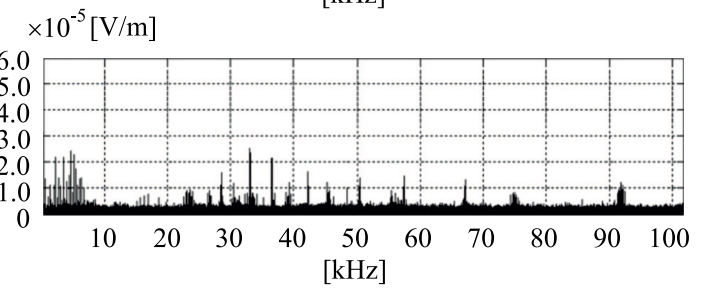

Fig. 3. Electromagnetic interference floor level without the shield: (a) $H$ component, b) $E$ component; and with the shield installed for $H$ and $E$, respectively.

(Fig. 3a-b). The floor noise for $E$ and $H$ field components was $10^{-5} \mathrm{~V} / \mathrm{m}$ and $10^{-5} \mathrm{~A} / \mathrm{m}$. After copper shielding, the interference was additionally reduced by the factor of two (Fig. 3c-d). Due to relatively high levels of EM fields generated by rocks subjected to the test, EM disturbances penetrating behind the screen are negligible.

\subsection{E Front End}

The $\mathrm{SEM}_{\mathrm{A}}$ electromotive force in a vertical sensor antenna with the height of $h=10 \mathrm{~cm}$ may be defined as [13], [14]:

$$
\mathrm{SEM}_{\mathrm{A}}=E \cdot h_{e f f}
$$

where: $E$ - electric field strength and $h_{e f f}=h / 2=5 \mathrm{~cm}$. The above relationship is true when condition [13] is fulfilled:

$$
h_{\text {eff }} \ll \lambda .
$$

In the frequency range of $0.1-100 \mathrm{kHz}$, the condition (6) may be considered fulfilled.

The signal from antenna is then amplified with the gain of $G u=96 \mathrm{~V} / \mathrm{V}$. The output voltage is:

$$
U_{\text {out }}=\mathrm{SEM}_{\mathrm{A}} \cdot G u=E \cdot h_{e f f} \cdot G u .
$$

Therefore, the value of $E$ field strength is determined by:

$$
E=\frac{U_{o u t}}{h_{e f f} \cdot G u}
$$

After amplification, the signal passes through a second order high-pass filter with the cut-off frequency of $f_{g r}=$

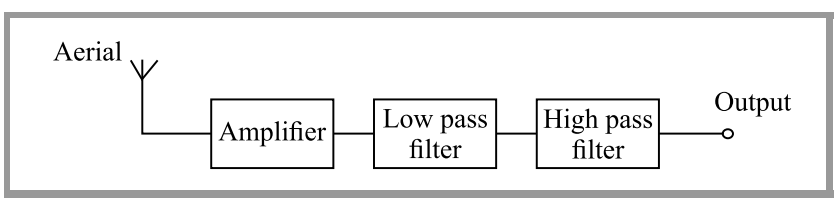

Fig. 4. Block diagram of an electric field front-end measurement unit.
$100 \mathrm{~Hz}$, and a second order low-pass filter with the limit of $f_{g r}=100$. According to Eq. (8), the output voltage of $10 \mathrm{mV}$ corresponds to the $E$ field strength of $2.1 \cdot 10^{-3} \mathrm{~V} / \mathrm{m}$.

\subsection{H Field Front End}

The magnetic field receiver (Fig. 5) utilizes a $100 \mathrm{~cm}$ long ferrite antenna with the diameter of $1 \mathrm{~cm}$. The coil comprises 900 windings of a $0.1 \mathrm{~mm}$ wire.

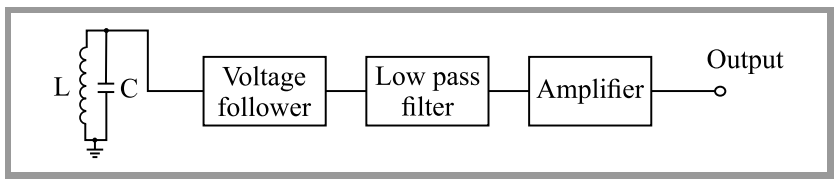

Fig. 5. Block diagram of a magnetic field front-end measurement unit.

The effective height of the ferrite antenna is [14]:

$$
h_{s k}=\frac{2 \pi z S \mu}{\lambda}=\frac{2 \pi f z S \mu}{c},
$$

where: $z$-number of antenna coils, $S$ - area covered by the antenna coil, $\lambda$ - wavelength, $\mu$ - magnetic permeability effective value of the antenna's ferrite core.

The $\mathrm{SEM}_{\mathrm{A}}$ electromotive force in the antenna coil can be determined from [14]:

$$
\mathrm{SEM}_{\mathrm{A}}=Z_{0} \cdot H \cdot h_{\text {eff }},
$$

where: $H$ - magnetic field strength, $Z_{0}=120 \pi \Omega$ - wave impedance of the free space.

The resonant frequency $f_{r}$ of the parallel LC antenna circuit (Fig. 5) is [15]:

$$
f_{r}=\frac{1}{2 \pi \sqrt{L \cdot C}},
$$

where: $L$ - inductance of antenna coil, $C$ - parallel antenna capacitance. 
Inductance of the ferrite coil can be calculated from [14]:

$$
L=\frac{z^{2} \cdot S \cdot \mu}{l}
$$

where: $z$ - number of windings, $S$ - area covered by the coil, $\mu$ - effective magnetic permeability of ferrite antenna core, $l$ - length of coil.

Below $f_{r}$ the effective antenna height $h_{s k}$ increases proportionally to the frequency level, with a slope of $20 \mathrm{~dB} / \mathrm{dec}$ [12]. In order to render the processing characteristics of the receiver as flat as possible, a first order low-pass filter with the cut-off frequency of $f_{g r}=96.5 \mathrm{kHz}$ was used, whose transmittance decreases proportionally to the frequency level, with a slope of $20 \mathrm{~dB} / \mathrm{dec}$ [13]. The amplifier gain is $K u=476 \mathrm{~V} / \mathrm{V}$.

Based on the measurements, it was found that the output voltage of $10 \mathrm{mV}$ corresponds to the magnetic field strength of $1.5 \cdot 10^{-5} \mathrm{~A} / \mathrm{m}$.

\section{Hard Coal Sample Measurements}

Several tests have been performed in which cube-shaped hard coal rock samples with the size of approximately $30 \mathrm{~mm}$ were destroyed. A linearly increasing force generated by the press was applied until the destruction of the tested sample occurred at the pressure of approximately $40 \mathrm{kN}$. Cube-shaped samples of coal, with their sides measuring $30 \mathrm{~mm}$, were examined.

Figure 6 shows the results for two representative coal samples. The $E$ and $H$ fields for two samples in the time domain are shown.

As a result of increasing force, the first micro-cracks appear in the sample even before it is destroyed. This phenomenon is noticeable on both electromagnetic field components, in the form of an initial, weak initiating pulse. The destruction process begins a moment later and is visible as a series of strong $E$ and $H$ pulses. The strength of $H$ field changes within the range of $\pm 0.1 \mathrm{~A} / \mathrm{m}$ range, while $E$ field varies by $\pm 1.5 \mathrm{~A} / \mathrm{m}$. The changes of both components have the (a)

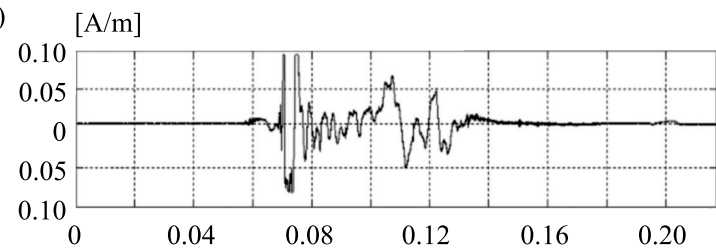

(c)

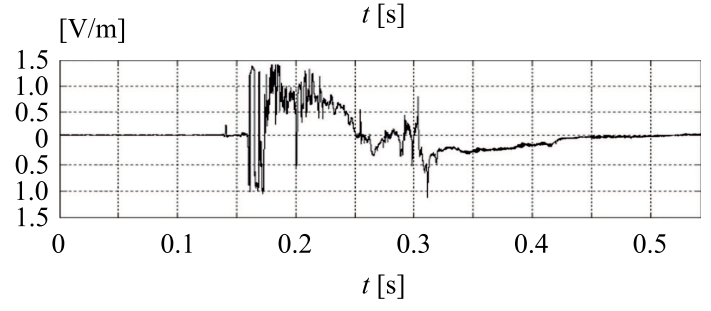

(b)

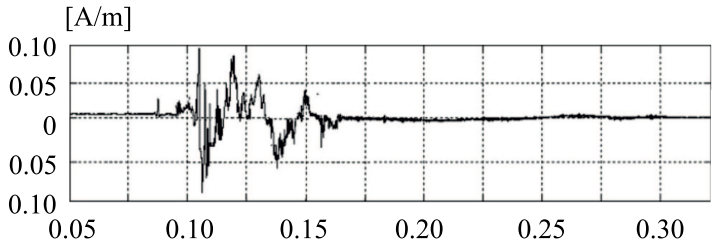

(d)

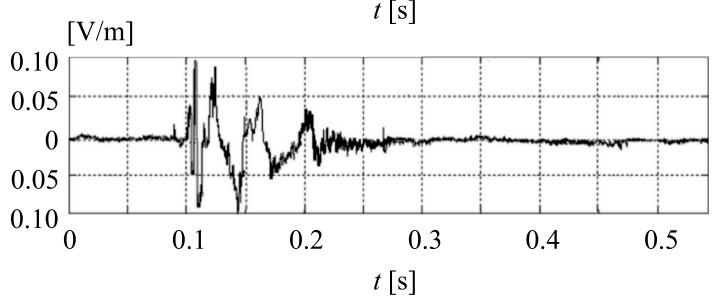

Fig. 6. Electromagnetic field variation over time during destruction of hard coal samples: (a) $H$ field, sample 1; (b) $H$ field, sample 2; (c) $E$ field, sample 1; (d) $E$ field, sample 2.

(a)

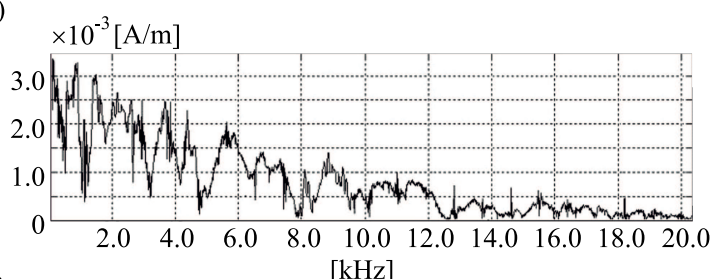

(c)

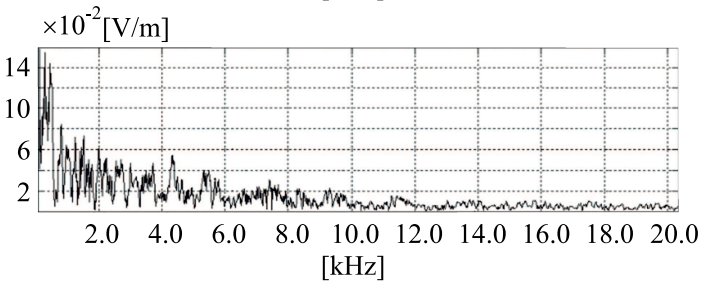

(b)

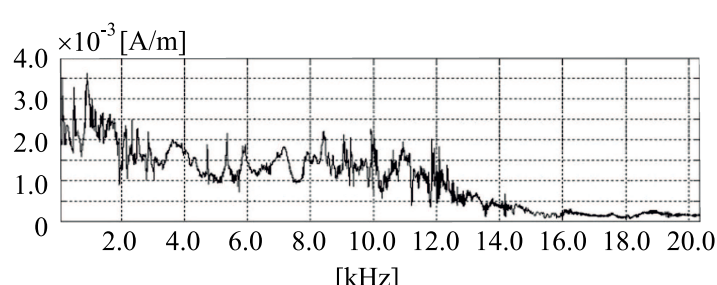

(d)

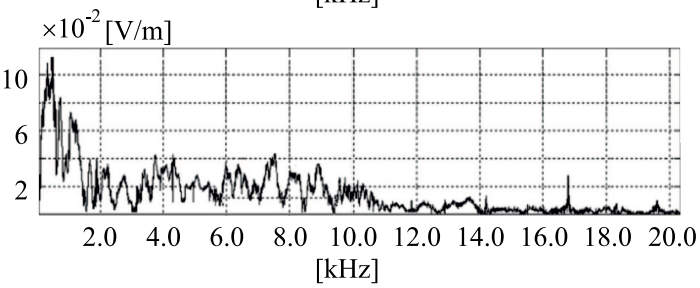

Fig. 7. Electromagnetic field variation in the frequency domain during destruction of hard coal samples: (a) $H$ field, sample 1; (b) $H$ field, sample 2; (c) $E$ field, sample 1; (d) $E$ field, sample 2. 
shape of decreasing pulses, which is well noticeable in the data concerning sample 2. The average duration of $H$ emissions for the tested samples was up to $100 \mathrm{~ms}$ for $H$ field and below $400 \mathrm{~ms}$ for $E$ field. Duration of the $E$ component is, on average, four times longer than that of the $H$ component.

In the frequency domain (Fig. 7), one may notice that the spectrum of the $H$ component is occupied up to $12 \mathrm{kHz}$, with peak from $1 \mathrm{~A} / \mathrm{m} \cdot 10^{-3} \mathrm{~A} / \mathrm{m}$ to $3.5 \cdot 10^{-3} \mathrm{~A} / \mathrm{m}$. For the $E$ component, the spectrum is occupied up to $2 \mathrm{kHz}$, and the peak values vary from 4 to $16 \cdot 10^{-2} \mathrm{~V} / \mathrm{m}$.

It is the formation of micro-cracks in hard coal caused by the force applied that is the primary source of EM emissions. The electrical component clearly interacts with the dynamics of the micro-fracture formation process. The electrical component is emitted already at the time of formation of the first micro-cracks, even before the initiation of the sample destruction process. It lasts throughout the entire crushing period, causing significant variations in field strength.

\section{Conclusions}

The measurements conducted confirm the existence of electromagnetic field emissions generated by rock material subjected to destructive mechanical stress. In the tested hard coal samples, this was observed both before the destruction and during the crushing process.

The increasing force results in peak EM field emissions, which can be considered one of the leading precursors of potential rock destruction. In mining, this can be related to the process of a potential collapse.

Time and frequency domain analysis allows to separate meaningful information from noise and makes it possible to design early warning systems informing about potential collapses.

In the future, the authors plan to build an autonomous EM field component recorder to be used in real mining conditions. The recorded data will provide an opportunity to perform comparisons with the results obtained with a network of geophones recording the seismic events occurring in mines, and will serve as a basis for evaluating practical usefulness of research concerning EM components emissions considered to be a precursor of seismic events.

\section{References}

[1] K. Holub, J. Holecko, J. Rusajova, and A. Dombkowa, "Long-term development of seismic monitoring networks in the Ostrava-Karviná coal mine district", Acta Geodynam. et Geomater., vol. 9, no. 2, pp. 115-132, 2012 [Online]. Available:

https://www.irsm.cas.cz/materialy/acta_content/

2012_02/1_Holub.pdf

[2] J. W. Warwick, C. Stoker, and T. R. Meyer, "Radio emission with rock fracture: possible application to the great Chilean earthquake of May 22", J. of Geophys. Res., vol. 87, no. B4, pp. 2851-2859, 1960 (doi: 10.1029/JB087iB04p02851).
[3] M. Hayakawa, T. Itoh, K. Hattori, and K. Yumoto, "ULF electromagnetic precursors for an earthquake at Biak, Indonesia on February 17, 1996", Geophys. Res. Lett., vol. 27, no. 10, pp. 1531-1534, 2000 (doi: 10.1029/1999GL005432).

[4] H. Greiling and H. Obermeyer, "Natural electromagnetic radiation (EMR) and its application in structural geology and neotectonics", J. Gelog. Soc. of India, vol. 75, pp. 278-288, 2010 (doi: 10.1007/s12594-010-0015-y).

[5] X. Li et al., "Rock burst monitoring by integrated microseismic and electromagnetic radiation methods", Rock Mechan. and Rock Engin., vol. 49, no. 11, pp. 4393-4406, 2016 (doi: 10.1007/s00603-016-1037-6).

[6] A. Jarosevic and J. Kundracik, "Measurement of the natural highfrequency magnetic field (PEE) in the boreholes using the FJV99 selective picoteslameter" EGRSE J., vol. 11, no. 1-2, pp. 8-12, 2004.

[7] S. Chen and E. Wang, "Electromagnetic radiation signals of coal or rock denoising based on morphological filter", Procedia Engin., vol. 26, pp. 588-594, 2011 (doi: 10.1016/j.proeng.2011.11.2210).

[8] M. Staworko and T. Uhl, "Modeling and simulation of piezoelectric elements - comparison of available methods and tools", $\mathrm{Me}$ chanics, vol. 27, no. 4, pp. 161-171, 2008 [Online]. Available: http://journals.bg.agh.edu.pl/MECHANICS/2008-04/mech05.pdf

[9] W. Wang, X. Xue, and J. Shan, "Theoretical models of seismic electromagnetic radiation based on piezoelectric effect", Indian J. of Geo-Marine Sci., vol. 44, pp. 1275-1281, 2015 [Online]. Available: http://nopr.niscair.res.in/bitstream/123456789/34906/1/ IJMS 44(9) 1275-1281.pdf

[10] S. Koshevaya et al., "Spectrum of the seismic-electromagnetic and acoustic waves caused by seismic and volcano activity", Natural Hazards and Earth Syst. Sci., vol. 5, no. 2, pp. 203-209, 2005 (doi: 10.5194/nhess-5-203-2005).

[11] E. Fedorov, V. Pilipenko, and S. Uyeda, "Electric and magnetic fields generated by electrokinetic processes in a conductive crust"; Phys. and Chem. of the Earth, vol. 26, no. 10-12, pp. 793-799, 2001 (doi: 10.1016/S1464-1917(01)95027-5).

[12] J. Carr, Practical Antenna Handbook. McGraw-Hill, 2001 (ISBN: 978-0071374354).

[13] U. Tietze and Ch. Schenk, Electronic Circuits. Handbook for Design and Application. Berlin, Heidelberg: Springer, 2008 (ISBN: 9783540786559).

[14] V. Dyo, T. Ajmal, B. Allen, D. Jazani, and I. Ivanov "Design of a ferrite rod antenna for harvesting energy from medium wave broadcast signals", The J. of Engin., vol. 2013, no. 12, pp. 89-96, 2013 (doi: 10.1049/joe.2013.0126).

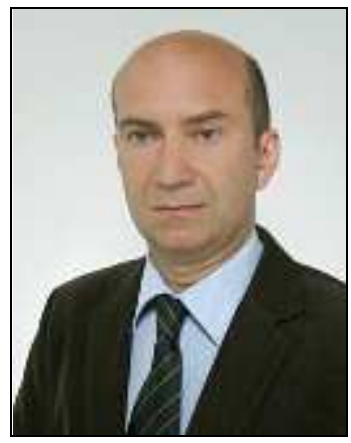

Remigiusz Mydlikowski received the M.Sc. and Ph.D. degrees in Electrical Engineering from the Wroclaw University of Science and Technology, Wrocław, Poland, in 1997 and 2002, respectively. $\mathrm{He}$ is currently in the Department of Theory of Field Theory, Electronic Systems and Optoelectronics at Wroclaw University of Science and Technology. His research interests are in non-invasive study of ground and rock properties.

(iD) https://orcid.org/0000-0002-8670-2913

E-mail: remigiusz.mydlikowski@pwr.edu.pl

Wroclaw University of Science and Technology

Wybrzeże Wyspiańskiego 27

50-370 Wrocław, Poland 


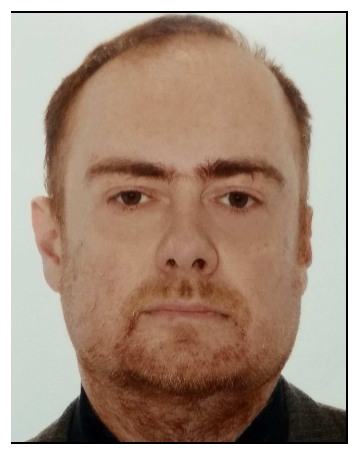

Krzysztof Maniak received his M.Sc. and Ph.D. degrees in Electronics from Wroclaw University of Technology, Poland, in 2001 and 2008, respectively. Since 2012, he has been with the National Institute of Telecommunications in Wrocław. He is an author and coauthor of numerous research papers and publications. His professional work focuses on: power systems, electromagnetic compatibility and design of measuring equipment. $\mathrm{He}$ also participates in research and measurement-related activities. His research interests focus on the testing of power grid quality and its broadband interference protection.

(D) https://orcid.org/0000-0003-1974-909X

E-mail: k.maniak@itl.waw.pl

National Institute of Telecommunications Swojczycka 38

51-501 Wrocław, Poland 